\title{
Designing Robots in the Wild: In situ Prototype Evaluation for a Break Management Robot
}

\author{
Selma Šabanović, \\ Indiana University Bloomington \\ Sarah M. Reeder, \\ Carnegie Mellon University \\ and \\ Bobak Kechavarzi \\ Indiana University-Purdue University Indianapolis
}

As robots move into everyday environments, we need to understand both the social and the technical constraints and affordances for human-robot interaction. We use in situ evaluation of partially functioning prototypes to inform the design of robotic technologies that fit their intended contexts of use and illustrate this method through a case study of iteratively designing a desktop robot for break management in a computerized office. After an initial exploratory study of the office as context of use, we used comparative semi-controlled evaluations of multiple design alternatives to explore how different robot characteristics, specifically embodiment and social interactivity, are perceived by users and affect their break taking. We found evaluating simple prototypes with varying levels of functionality, even when not robust or "complete," provides opportunities for including users in the design process and for identifying emergent factors that impact robot use. Our case study provides insights into the challenges and best practices for performing iterative prototyping and in situ evaluations of robots, which can inform future development of contextually appropriate robotic technologies.

Keywords: human-robot interaction, in situ evaluation, prototyping, social and assistive robots, design methods, socially situated robots

\section{Introduction}

Robots used outside of controlled environments, such as laboratories and factories, often have unexpected contextual effects that challenge initial design assumptions and can determine the eventual success or failure of robotic applications. In the context of users' homes, utilitarian robotic vacuum cleaners were treated as "social products" (Forlizzi, 2007). In a hospital, an assistive robot lauded by management and accepted by postpartum staff was seen as a nuisance by oncology staff (Mutlu \& Forlizzi, 2008). Conference attendees encountering a robot seeking their assistance were more likely to ignore the robot in a utilitarian hallway and to respond to its pleas in a social reception (Michalowski et al., 2007). These examples suggest that developing robots for use in everyday social contexts, such as in private homes (e.g., Gates, 2007; Lee et al., 2012), healthcare institutions (e.g., Broekens, Heerink, \& Rosendal, 2009; Inoue et al., 2010; Mutlu \& Forlizzi, 2008;), shopping malls (e.g., Kanda et al., 2009), museums (e.g., Nomura et al., 2007), classrooms (e.g., Kanda et al., 2007; Tanaka et al., 2007) and offices, requires research, design,

Authors retain copyright and grant the Journal of Human-Robot Interaction right of first publication with the work simultaneously licensed under a Creative Commons Attribution License that allows others to share the work with an acknowledgement of the work's authorship and initial publication in this journal.

Journal of Human-Robot Interaction, Vol. 3, No. 1, 2014, Pages 70-88, DOI 10.5898/JHRI.3.1.Sabanovic 
and evaluation that address the emergent situated interaction dynamics between people and robots in specific contexts of use.

Although uniquely suited to exploring the situated dynamics of HRI, studies outside the laboratory represent a small subset within human-robot interaction (HRI) research. The more predominant controlled lab studies are not conducive to identifying contextual effects that emerge in open-ended encounters between humans and robots (Šabanović, Michalowski, \& Simmons, 2006). Human-Computer Interaction (HCI) researchers have also suggested that theories about interaction produced in the laboratory do not precisely map onto the real world (Rogers, 2011) and are of limited value for understanding how users will respond to technologies in their daily lives. $\mathrm{HCI}$ researchers often use partially functioning prototypes to test designs in their intended contexts of use early in the development process, when insights from the studies can be most influential in determining the functionality of the final product (Gould \& Lewis, 1985). Inspired by these approaches, we present a case study of the iterative prototyping and evaluation of a break management robot as an example of a socially situated approach to the design of robotic applications for everyday use that recognizes the mutual shaping of the social and technical aspects of robotics (Šabanović, 2010).

The presented case study describes design-oriented research exploration in HRI through in situ evaluation and parallel comparisons of multiple design alternatives to understand how specific parameters of a robot under construction (e.g., embodiment, social interactivity) drive its performance in the field. Participants' experiences, reactions to, and critiques of our prototypes guided the choice of problems and research questions in more contextually appropriate directions, helping us evaluate specific interaction design components and produce further ideas for iterative development of the robot. From ideation through two material instantiations, our experiences illustrate the challenges and valuable insights that can be gained by testing robotic technologies in the prototype stage in their intended contexts of use. In the discussion, we generalize from our work to discuss limitations and useful aspects of our method of iterative in situ prototype development and evaluation for HRI research and design. Our research suggests that evaluating HRI in the field early and often can produce the contextual knowledge needed for developing socially appropriate robotic technologies.

\section{Background/Motivation}

The approach discussed in this paper is grounded in an understanding of HRI design and use as socially situated practices, developing through the dynamic interaction of humans, robots, and the social spaces they co-inhabit while being inspired by the practices of prototyping and in situ evaluation in HCI and HRI.

\section{Socially Situating HRI Research and Design}

Technology and society are in a continuous process of co-evolution-social factors affect technology design and use, while the affordances and capabilities of technology have consequences for social organization, beliefs, and practices (e.g., Bijker, 1995; Nye, 2007). This mutual shaping of the technical and the social is often not taken into account in the process of designing robotic technologies, which envisions the social "impact" of robotic technologies as the result of their technical capabilities and disregards the role of the social context and diverse stakeholders, including users as well as technologists, in the success or failure of robotic products (Šabanović, 2010). Suchman (2007) critiques contemporary social robotics research for foregrounding the technical and "erasing the social"- the networks of human and non-human social agents, values, norms, frameworks, and practices that are the context for robotic development and their implementation into society. Discussing information and communication technologies more generally, Hakken, Teli, and D'Andrea (2010) suggest a lack of attention to the 
social in technology design and implementation has led to low rates of success in societal applications, particularly in cases where "technical virtuosity" is the dominant guiding value, as is commonly the case in robotics. They further suggest that developing more socially robust technologies requires that social and technical aspects are constructed in a symmetrical manner throughout the process of technology design and implementation (Hakken, Teli, \& D'Andrea, 2010). We posit that one way to take into account how technology is co-defined with its sociocultural environment is to approach robot development in an exploratory manner through in situ evaluations of multiple prototypes of possible robotic technologies. Rogers (2011) suggests that such a process of "design in the wild," where technologies and artifacts are constructed and then tested in the expected context of use, is necessary to show "how people come to understand and appropriate technologies on their own terms and for their own situated purposes" (p. 59).

\section{In Situ Evaluation of Emerging Robotic Technologies}

Studies evaluating interactions between people and robots in various contexts of use, including hospitals (e.g., Mutlu \& Forlizzi, 2008), other healthcare institutions (e.g., Chang, Šabanović, \& Huber, 2013; Kidd, Taggart, \& Turkle, 2006; Wada \& Shibata, 2007), and private homes (e.g., Forlizzi, 2007; Forlizzi, DiSalvo, \& Gemperle, 2004; Sung, Christensen, \& Grinter, 2009), have brought attention to the mutual shaping of the technical and social in HRI, the relational nature of social agency and autonomy ascribed to robots, and to the effects of emergent social and cultural factors on the success and consequences of robotic applications in society. Morana Alač's (2011) observational studies of HRI research in a nursery school show that the social agency of robots is constructed not through their inherent capabilities alone, but with regard to the social behaviors of people in their environment. Wada and Shibata's (2007) research on the use of the seal-like robot PARO in nursing homes discovered that the robot acted as a social mediator, facilitating interaction among nursing home residents. Chang, Šabanović, \& Huber's (2013) observational study of open-ended interactions between nursing home residents, staff, and visitors and the robot PARO show that the mediation of therapists, participants' individual interpretations of PARO, and the social environment have significant effects on the success of people's interactions with the robot. Studies of Roombas in the home (Forlizzi, 2007) suggest that the robot's introduction affected the social organization and meaning of cleaning, while the social nature of the home reflected on the users' interpretations of the robot as a social product. Providing an organizational perspective on HRI, Vertesi's (2012) ethnographic studies of NASA's Mars Rover missions display how the function and meaning of a robotic artifact is supported through specific and deliberate forms of social organization as well as participants' embodied identification with the robot's functions. Such studies have been invaluable for understanding the ongoing construction of meaning and practice in interactions between humans and robots that influence use and can be a resource for design.

\section{Prototyping in HCI and HRI}

While in situ evaluation can help us understand the socially situated dynamics of using existing technologies, the various prototyping and evaluation practices employed in $\mathrm{HCI}$ can be useful models for design-oriented research and exploration in HRI. HCI researchers use prototypes to evaluate and generate design ideas, provide a focus for exploration, learning, and refining designs and their initial assumptions, while helping to conceptualize a variety of different dimensions of an artifact-its appearance, functionality, interactivity, and spatial structure (Lim \& Stolterman, 2008). They can also serve as "vehicles for communication" in ways that include users in the early stages of design (Holmquist, 2005); the unfinished nature of prototypes has been found to inspire more input from users (Rettig, 1994). Prototypes can also help researchers develop an understanding of the experience of interacting with a technology (Buchenau \& Suri, 2000), emphasizing that even simple artifacts are experienced in the context of dynamic and shifting relationships with other people, places, things, and ideas. In general, the development and 
testing of physical prototypes helps designers model agent-environment interactions (Bird, Marshall, \& Rogers, 2009). Furthermore, the production and testing of multiple design ideas at the same time, which Dow et al. $(2010,2011)$ called "parallel prototyping," not only creates more successful products but also promotes collaboration and encourages exploration among participants in the design process without investing in one idea too early.

Prototyping has been less prevalent in HRI due to the challenge of quickly constructing diverse, temporary, yet robust and capable platforms (e.g., Bartneck \& Hu, 2004; Lee et al., 2009; Reeder et al., 2010; Šabanovic et al., 2011). The most well known prototyping technique in HRI is Wizard of $\mathrm{Oz}(\mathrm{WoZ})$, in which autonomous robotic behavior is simulated by a person controlling the robot with the aim of measuring user reactions (Dautenhahn, 2007; Riek, 2012). Lee et al. (2009) used the WoZ technique iteratively in designing the Snackbot platform. HRI researchers have also experimented with other ways of prototyping and testing HRI in context. Michalowski et al. (2006) used 3D animations and a puppet to stand in for a functioning robot during early behavioral design. Bartneck and Hu (2004) used rapid prototyping of robot embodiments with Lego Mindstorms and simple electronics to incorporate observations from user and environment interactions early on in the HRI design process. Prototyping has also been used in participatory approaches to robot design (e.g., DiSalvo et al., 2008; DiSalvo et al., 2011) to allow users to provide engaged and situated input in the design and application of new robotic technologies in society.

More recently, electronic prototyping platforms, such as Arduino microcontroller kits (http://arduino.cc/), the mbed rapid prototyping platform (mbed.org), and the Raspberry Pi computer (http://www.raspberrypi.org/) have extended the ability of researchers and designers to evaluate their interactive technology ideas without expensive fully functional prototypes. These platforms provide new opportunities for developing a more iterative and socially situated process of HRI design through prototyping and in situ evaluation.

\section{Dewey: A Case Study of In Situ Evaluation of an HRI Prototype}

The following case study of iterative prototyping and in situ evaluation of a break management robot developed for the computerized office presents a methodology for developing a socially situated understanding of how particular design characteristics of robots affect people's responses and sense making within specific contexts of use. The project was motivated by an established problem in the contemporary workplace: the need to take regular breaks during the workday to prevent injury and alleviate fatigue that can have negative effects on health and job performance (Jett \& George, 2003). Existing technological interventions in this domain include various computer applications using verbal, audio, or visual cues to remind people to take a break (e.g., Morris, Brush, \& Meyers, 2008), as well as the Breakaway prototype (Jafarinaimi et al., 2005), an embodied ambient display for break management. We provide more information on existing break management technologies and their comparison with our system in prior work (Reeder et al., 2010; Šabanović et al., 2011). Research on socially assistive robotics (e.g., Kidd \& Breazeal, 2008; Tapus, Matarić, \& Scassellati, 2007) suggests that embodied and socially interactive technologies can have stronger positive effects on behavioral change and compliance in users than computerbased technologies. We therefore decided to design and evaluate a robotic break management prototype for the office and performed three iterative design studies exploring the context of use and user needs and reactions to robotic technologies. We refer to our series of robotic prototypes as "Dewey" to signify their do-it-yourself (DIY) nature.

All three studies were performed with staff members at Indiana University, Bloomington, who engaged in regular computer use for 6 or more hours a day throughout the workweek. We first performed an exploratory study of the office, which allowed us to document the significance of break management for our population, learn more about the work environment, and evaluate a non-functional physical prototype of a break management robot (Reeder et al., 2010). The following two studies involved the development and testing of two functioning physical 
prototypes in regards to how users would react to and make sense of specific aspects of the robot's design and to further explore use in context. All design iterations were based on findings from previous studies; in iterations 2 and 3 we followed a comparative approach of evaluating multiple versions of our technology at the same time, consistent with Dow et al.'s $(2010,2011)$ notion of "parallel prototyping," to understand the contextual effects and meanings of particular aspects of the robot's design (i.e., embodiment, social interaction). Our main aims were two-fold: to identify the appropriate design characteristics for a robot that can help people manage their breaks in daily use and to explore the dynamics of open-ended HRI in natural human environments. Through this process, we also gained insights about the evaluation of HRI prototypes "in the wild" as a way to contribute to our understanding of the socially situated dynamics of HRI that can be a resource for further design. As the details of the first two studies have been published previously (Reeder et al., 2010; Šabanović et al., 2011), we summarize relevant insights here and provide a more detailed account of the third iteration of our break management prototype. Table 1 summarizes the main aims and findings, while Figure 2 shows the timelines of the three design iterations.

Table 1. A summary of the main aims and findings of the three Dewey design iterations.

\begin{tabular}{|c|c|c|}
\hline Design Iteration & Aims & Findings \\
\hline $\begin{array}{c}\text { 1: Context } \\
\text { exploration }\end{array}$ & $\begin{array}{l}\text { Exploration of taking } \\
\text { breaks in an office } \\
\text { environment and initial } \\
\text { design of a physical break } \\
\text { reminder technology. }\end{array}$ & $\begin{array}{l}\text { Minimalist design preferred; } \\
\text { users concerned about amount of } \\
\text { effort involved in using } \\
\text { technology; desire for small } \\
\text { footprint and no noise. }\end{array}$ \\
\hline 2: Embodiment & $\begin{array}{l}\text { Comparison of physically } \\
\text { embodied and virtual } \\
\text { prototypes in respect to } \\
\text { break-taking behaviors. }\end{array}$ & $\begin{array}{l}\text { Embodied prototype preferred } \\
\text { and more efficient at reminding } \\
\text { about breaks; users requested } \\
\text { more interactivity and } \\
\text { personalization. }\end{array}$ \\
\hline 3: Social interaction & $\begin{array}{l}\text { Examination of how } \\
\text { social interactivity affects } \\
\text { perception of the robot } \\
\text { and effectiveness in break } \\
\text { management. }\end{array}$ & $\begin{array}{l}\text { Social robots are described in } \\
\text { more relational terms and more } \\
\text { effective in motivating taking } \\
\text { breaks; work style has } \\
\text { significant effect on perceptions } \\
\text { of robots. }\end{array}$ \\
\hline \multicolumn{2}{|c}{} & \\
\hline
\end{tabular}

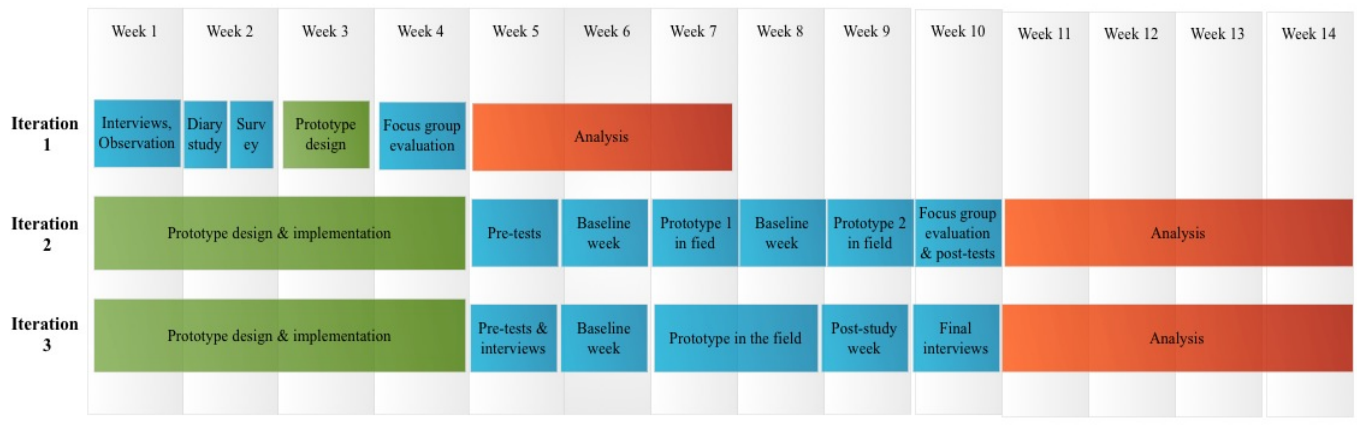

Figure 1. A timeline of our three design evaluations, showing the time needed to develop the prototype, perform design and evaluation research in our field sites, and analyze the collected data to inform further iterations. 


\section{Design Iteration 1: Learning From the Context of Use}

Our first iteration aimed to study the organization of work in a computerized office, including people's daily break-taking habits and workflow, while testing out our initial ideas for the form and functions of a break management robot. We interviewed six volunteer participants about their break-taking practices and found they varied widely, but dissatisfaction with the frequency of breaks was commonplace. A diary study with three other participants, during which they carried a small notebook for a day and used it to log the timing and reasons for their breaks, helped us to understand how, why, and when participants took breaks as well as to gauge how willing users might be to carry a robot with them throughout the day. The study suggested that most breaks tended to last a few minutes, were irregularly spaced, and often taken to perform work-related tasks or tend to physical needs. Participants did not consistently carry the logbook with them, suggesting we should not rely on them to carry the robot around. We ended our initial exploration by surveying 16 members of the University community about their preferences for possible forms for the robot, ranging from minimalist to creature-like, noting a positive attitude towards minimally designed robots.

The insights from our initial exploration of the office environment allowed us to develop an ideal "use scenario" and the first physical prototype for our robot. The prototype was creature-like in form and used minimalist design cues (e.g., Bethel \& Murphy, 2008; Kozima, Michalowski, \& Nakagawa, 2009), such as rocking and light, to communicate with users (see Figure 2). Our use scenario envisioned the robot belonging to one user who would keep it on their desk in the office. The robot would vibrate and blink its lights at regular intervals throughout the day to signal to the user that they should take a break. We built the prototype using an Arduino microcontroller wired to a tricolor LED and vibration and servo motors to enable movement. The body was constructed using Model Magic and included fiberoptic tufts that were not functional. Though the initial prototype did not have full functionality, it was useful as an "idea generator" in our dicussions with potential users. In a focus group, we described the different components and design of the prototype to the participants, showed them the physical form, and demonstrated the rocking behavior that would alert them about breaks. This inspired participants to discuss the robot's size, particularly its footprint on their desk, lowering the noise made by the motors, and possibilities for personalization to fit a user's mood or be more distinctive. Users also showed concern about being responsible for the robot, which suggested effort in operating the robot should be minimal. The overall feedback was positive and suggested a willingness to use the robot. These initial exploratory studies of the context of use and initial feedback on our prototype allowed us to ground further studies and design in the experiences and stated preferences of our target users.
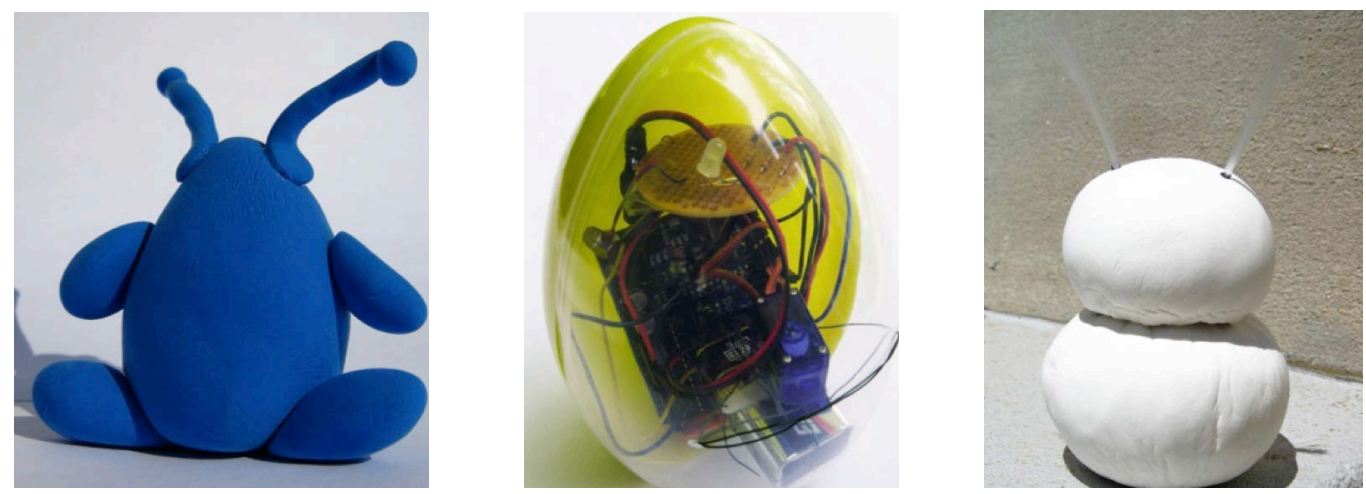

Figure 2. A sample of a creature-like form for the robot presented to users, the functioning robotic prototype, and the Model Magic shell with fiber optic tufts. 


\section{Design Iteration 2: Evaluating Embodiment}

Our initial study suggested that users would be interested in using a break management robot. As our next step, we wanted to validate the need for an embodied robotic technology, since they are more difficult to implement than the computer-based applications representing the majority of existing break management technologies. We also wanted to explore what techniques a robot might employ to be effective for break management through discussions with users after they had experienced the robot in their everyday work routines for some time. We developed two versions of our break management technology - a robot that could sit on a user's desk and a computer application that showed the same robot in a pop-up window - that we evaluated comparatively with the help of staff members in another University office. In line with the findings from our first evaluation, this second robot had a minimalist creature-like appearance (see Figure 3) and used nonverbal cues to interact with users. We wanted to test a functioning prototype this time and developed a robot that had four simple behaviors: bobbing side to side to greet the user; twisting its head as if looking around as the first break signal; faster head twisting as the second signal if the user does not take a break the first time; and even faster bobbing side to side repeated every 20 minutes until the user takes a break as the third break signal. The online version consisted of popup videos of the robot performing the four basic motions according to the interaction rules used by the embodied version. Physical prototypes were created using an Arduino microcontroller wired to appropriate sensors and servos, and repurposed materials purchased in a local department store for the robot's body. As our prior study suggested that users will not carry the robot with them on breaks, we designed a sensor packet using a second Arduino microcontroller connected to a noncontact temperature sensor and a passive infrared motion sensor to measure when the user was at their desk or away, with the latter used as a heuristic for break taking.
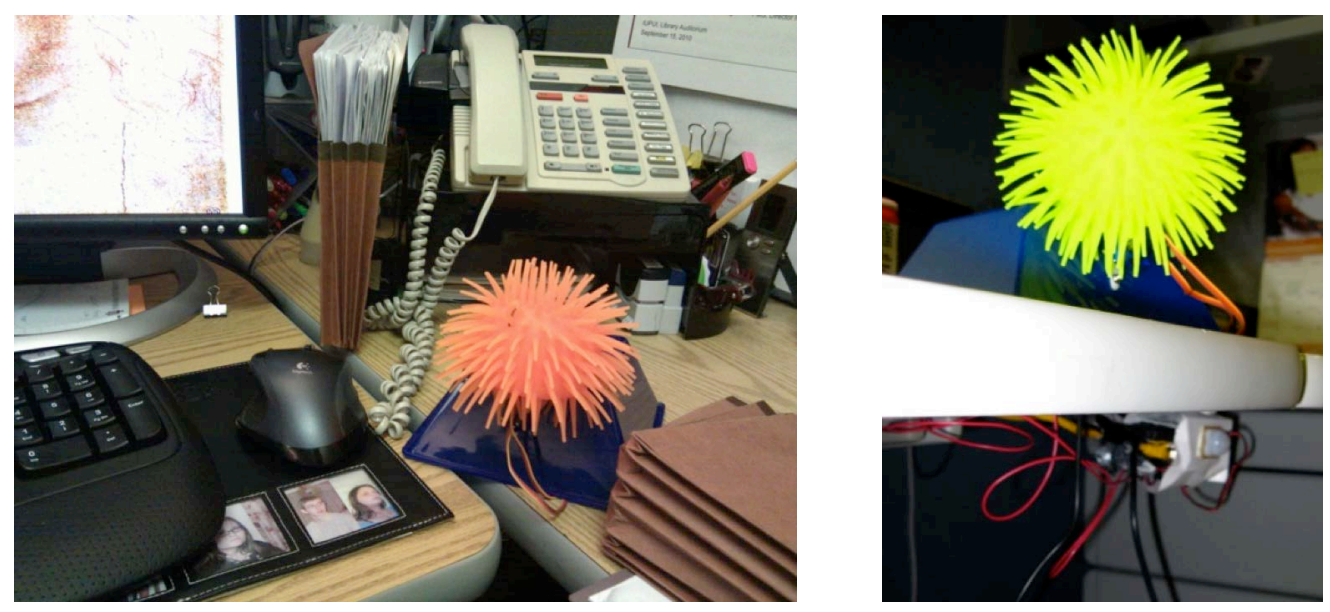

Figure 3. The second prototype on a user's desk during the field study; the prototype with the sensor packet mounted underneath the user's desk.

The user evaluation of our second prototype was performed with six participants (one male and five female), each of whom used both the robot and the computer agent for one week at a time. The study lasted a total of four weeks, with the first week establishing a baseline for break-taking behavior, the second week introducing one intervention, the third week establishing another baseline, and the fourth week introducing the second intervention. We took multiple measurements during the study, including sensor readouts of movement and temperature to establish participants' presence at their desks; pre- and post- interviews with participants about their break taking, work practices, and experiences with the technology; daily self-report surveys on participants' stress, productivity, and fatigue levels; and a final focus group with all 
participants where we compared the two technologies and discussed general ideas about designing break management applications.

This iteration not only helped us develop further design ideas for our robot, but it also gave us an appreciation for the challenges involved in evaluating functional physical prototypes of robots in natural use contexts. We experienced technical difficulties relating to the operation of the wireless network, which interrupted communication between the sensors and the robot and led to data loss or the inability to effectively communicate break suggestions. These failures required us to visit the field site repeatedly to restart the prototype or reattach sensor hardware, which in turn led the users to doubt the reliability of the system. Finally, a change in the University's security protocols made wireless communication with our sensors impossible and regulations forbade us from logging data locally on users' computers, so the study was ended after the first week of interventions.

Despite issues with study implementation, our access to users and their experience of having the prototypes in their offices allowed us to gather feedback that was useful for guiding further iterations. The limited break data that we managed to collect suggested that participants who interacted with the embodied robot experienced an increase in break frequencies, while those with the computer-based intervention did not (Šabanović et al., 2011). After a full demonstration of both versions of the robot in a focus group we organized at the end of the study, five out of six participants said that they preferred the embodied to the computer-based version, particularly emphasizing that its physicality afforded interaction and play, and was an unobtrusive yet constant reminder of the need to take regular breaks. While participants enjoyed the robot's simplicity because it meant they did not need to do anything extra to make it work, they also expressed a desire for more interactivity and feedback so they could understand what the robot was sensing and where they were in the break cycle. Participants also asked for the ability to control the robot by being able to turn it on and off, set up individual break preferences, and hit "snooze."

Our second study established the need for continuing to develop an embodied technology for break management. We also gained important insights into the technical and methodological implementation of field studies of robotic prototypes. One was to keep our technology as selfsufficient as possible, as our design had relied on too many elements (e.g., communication over the wireless network) that were out of our control. Our experience also suggested that field evaluations should involve the collection of many different kinds of data. While some of our data was unreliable (e.g., self-reports on break taking) and other methods of collection (e.g., direct observation or video recordings) were not available due to the participants' wishes, triangulation of different sources allowed us to get an understanding of the daily flow of activities of our users. The subjective reports on experiences of the users were the most helpful in developing further design iterations. The behavioral results allowed us to confirm the utility of an embodied break management technology, and user reports suggested that a more interactive and social interface should be the focus of our next design iteration.

\section{Design Iteration 3: Constructing Robotic Sociality}

Motivated by participant requests for a more interactive robot and by their reports of playful interactions with the prototypes, our main aim in the third iteration of design and evaluation was to explore how the robot's social interactivity would influence effectiveness in break management and user perceptions. We recruited nine participants, eight of whom were female and one male. Similarly to the prior study, this user evaluation was designed as a comparison between two different design ideas for our technology - one that functioned as a simple alarm and one with social behaviors - to gauge the effects of social interactivity on HRI for break management. The comparative methodology allowed us to pinpoint which user reactions were based on the robot's social capabilities. Each participant was given a robot to use for two weeks; half the participants received a socially interactive prototype and the others received the alarm. 
Robot Design and Use Scenario

The prototype design and daily interaction scenario in this iteration were inspired by insights from the previous two studies. The robot was once again envisioned as an artifact that users would keep on their desktop. At the start of their workday, participants indicated they were present by tapping the casing of the robot; the tapping was sensed by the piezoelectric sensor and switched the robot on. The robot then moved its head up from a resting position while the tricolor LED turned green to indicate that the system was working. After a preprogrammed interval-we chose 40 minutes according to prior research - the robot started turning its head slowly from left to right while the LED turned red to indicate the first alert to take a break. If the participant wished to comply with a break suggestion, they swiped an RFID card to indicate they were leaving, and when they returned they needed to swipe a different RFID card. Using RFID cards to signify when users were taking a break made the system more self-sufficient, in contrast to the unsuccessful networked design from iteration 2. If the participant did not wish to take a break, they ignored the suggestion. After a set period, the robot repeated its animated dance two more times. If the robot did not detect interaction after three suggestions, it entered a sleep state until the participant reset it by tapping on the casing.

We constructed ten prototypes; each used an Arduino Duemilanove board as the controller, an RFID receiver as a means for users to indicate their breaks to the robot, a piezoelectric sensor to sense when users tapped on the robot's casing or petted it, and a tricolor LED as a visual aid to indicate the state of the robot (see Figure 4). Two servomotors allowed the robot to turn its head on its axis and rock side to side. The robot recorded when participants took breaks and its own changing states in a text file that was stored in a folder on the participant's computer.

The alarm-type and social robots looked identical and functioned similarly with small variations. The social robot responded to "petting" and exhibited three different levels of agitation through increasingly vigorous movements. The alarm robot had only one behavior that acted as an alarm and did not exhibit different levels of agitation for successive break alerts. To differentiate the socially interactive robot from the alarm robot, we applied Turkle's (2006) idea that people will nurture robots to motivate participants; a participant in iteration 2 had also mentioned nurturing might encourage taking breaks. Nurturing was operationalized as the user "feeding" the robot RFID cards decorated to look like fruits (see Figure 5).

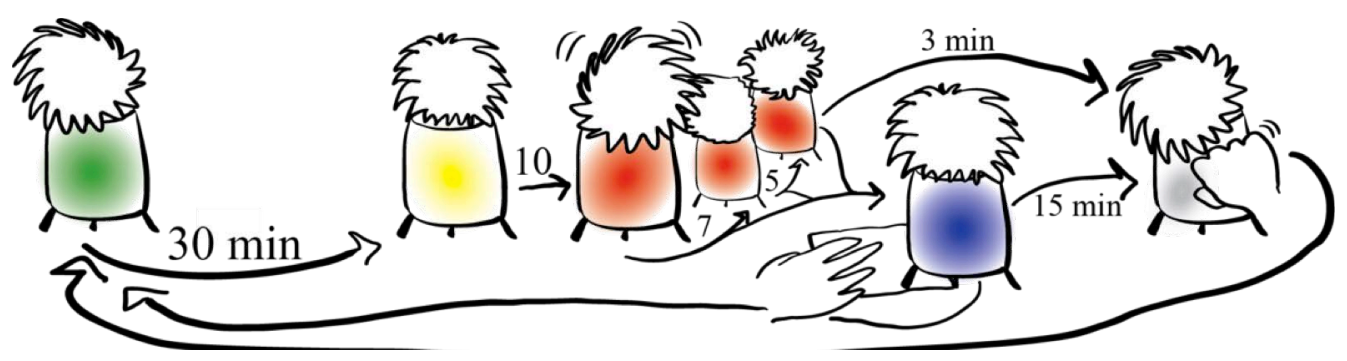

Figure 4. Dewey state transitions in break alerts. 

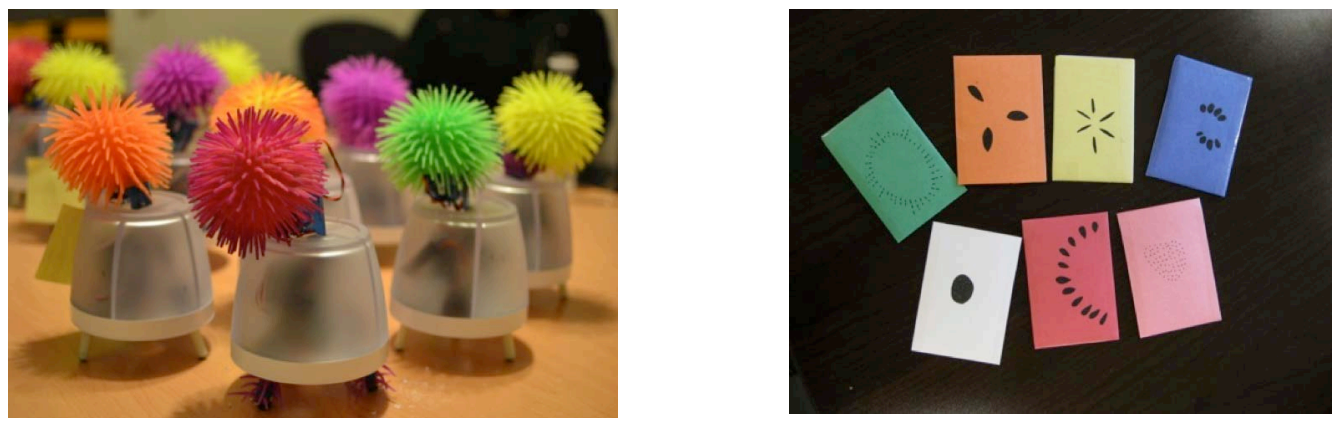

Figure 5. Dewey robot prototypes and RFID cards disguised as "fruit."

\section{Evaluation Process}

The user evaluation took place over four consecutive weeks. The first week established a baseline for the break-taking behavior of participants through self-report; the robots were introduced as a technological intervention in the second and third weeks, during which time we collected behavioral self-reports from users and logs recorded by the robots; in the final week, users selfreported break behaviors after the robots were removed. Four people received an alarm robot and five people received a social robot. The conditions were not randomly assigned; we assumed that participants who worked in close proximity would talk to each other about the project, so we gave all participants working in one building social robots (P1, P2, P4, P6, P10) and all participants in another building alarm robots (P5, P7, P8, P9). Work environments in the two locations were comparable.

Our study aimed to explore how social interactivity and other emergent factors affected participants' perceptions of the robots, their break-taking behavior, and their willingness to adopt the robot for future use. Before the study began, subjects participated in a semi-structured interview about their break taking, work practices, and attitudes towards technology and then filled out an online questionnaire. The questionnaire included items adapted from the "Technology Attitude Instrument" (Liu \& Maddux, 2004) and "Perceived Usefulness" scale (Davis, 1993), and the full "Negative Attitudes Towards Robots Scale" (NARS) (Nomura, Kanda, \& Suzuki, 2006) and "Emotional Contagion Scale" (Doherty, 1997). For the duration of the study, daily emails asked participants to report their perceived productivity, social and mental activity, fatigue, and stress using a 4-point scale ranging from "not at all" to "extremely," as well as the number of breaks they took with and without the robot's prompting. In the second week, participants received a break robot prototype that was one of two types: alarm or social. The participants did not know there were two types of robots and the two groups received different instructions for use. Recipients of the alarm robot were told they would get an alert when they needed to take a break; the directions for use were couched in technical terms regarding logging in and out with RFID cards. Recipients of the social robot were directed to "feed" the robot and told what conditions make it "happy." The RFID cards for the social robot were minimally decorated to symbolize different fruits, while the alarm robot cards were decorated to look like playing cards, allowing for easy identification without connoting animacy. Participants in both groups kept their robots for two weeks (10 work days). At the end of the fourth week, we asked participants to fill out an online exit survey adapted from the "Adoption of Information Technology in the Workplace" (Moore \& Benbasat, 1991) instrument, which included questions about voluntariness of future use, perceived relative advantage, compatibility, image, and ease of use. Researchers also met with participants for a final interview, which started with a modified version of Microsoft's Product 
Reaction Cards ${ }^{1}$ exercise and continued with questions about users' perceptions of the robot and their break-taking experiences during the study.

\section{Findings}

Our study produced multiple sources of data, but certain data sources again turned out to be unreliable, while the small number of participants allowed us to look for general trends rather than statistical significance in the data. Daily self-report data was inconclusive and did not display any differences among participants or the phases of the study. Participant responses during the final interviews suggest their stress, productivity, etc. had more to do with their daily work situation than the robot's behavior; some participants also did not fill out the survey that was emailed to them until the following morning and may not have remembered their state from the previous evening. We also saw little congruency between participants' self-reports of break-taking behavior and breaks logged by the robots, reconfirming results of previous studies that participant reports of behaviors often do not match sensor data (Hazlewood, Stolterman, \& Connelly, 2011). Post-study surveys, filled out by eight of the nine participants, showed both groups scoring towards the lower end to the middle of the scale, suggesting they were overall not very pleased with the robot. In final interviews, we learned their dissatisfaction largely stemmed from technical problems with the prototype rather than the design factors that we were interested in evaluating. The most technically savvy participant was more tolerant of breakdowns in the technology, ascribing them to the nature of prototypes in the final interview, which suggests it might be important to educate participants on prototypes as unfinished artifacts before the evaluation and to prompt them to reflect on the further development and possibilities for using a more robust version of the technology.

Robot behavior logs gave us more insight into the different ways in which participants responded to the two types of robots. We used logs for six participants in the analysis; we had to remove three because of technical problems in data collection. We noticed a difference across conditions in terms of when users responded to the robot's alerts. Users who had the alarm-type robot $(n=3)$ received a total of 86 alerts from the robot during the two weeks of use and responded most often to the first alert (27.9 percent of responses), followed by the second (9.3 percent) and third alerts ( 3.4 percent), and ignored 59.3 percent of the robot's total break alerts. Users with the social robot $(n=3)$ received a total of 118 break alerts during the two-week period and responded most often to the second alert (29.7 percent of responses), than to the first alert (22.9 percent of responses), and rarely let the robot go to the third alert (13 percent of responses), while ignoring only 34.7 percent of the robot's suggestions to take a break. The lower number of alerts presented to participants in the alarm group suggest they used the robot less; P7 and P9 corroborated this in their interviews, saying they did not "wake up" their robot when it was interfering with their work. The results suggest that the social robots were generally more effective in helping users manage breaks (see Figure 6a).
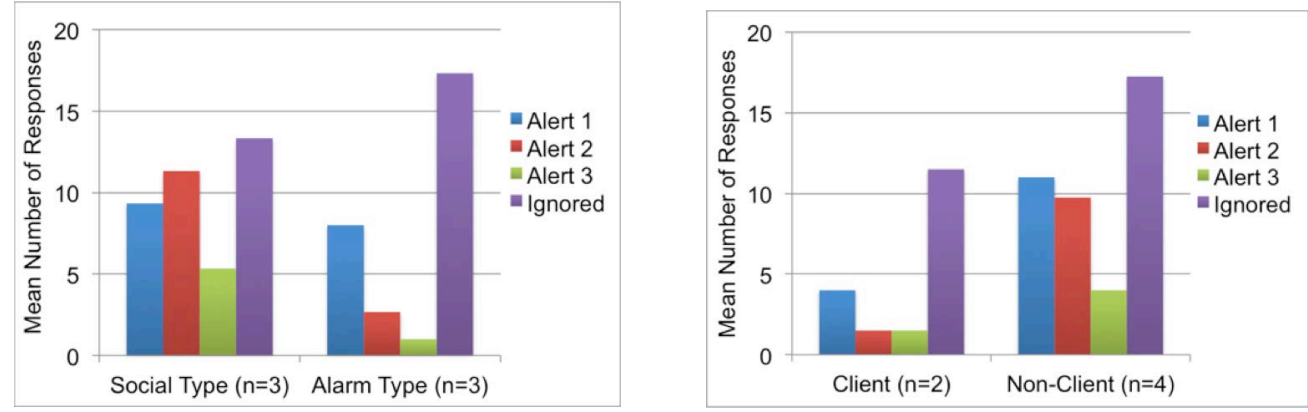

Figure 6. Participant responses according to (a) robot type and (b) work environment

\footnotetext{
${ }^{1}$ The procedure and the full set of cards are available at http://www.microsoft.com/usability/UEPostings/ProductReactionCards.doc
} 
The most compelling and useful information came from semi-structured interviews with participants at the end of the study. Interviews helped us identify the effect of a factor we had not considered, namely the participants' work style-defined as contact with clients and the regularity of interruptions - on the robot's efficacy and user perceptions. Five out of nine participants reported that their jobs involved a significant amount of interaction with other people (e.g., clients, students), while the remaining four participants said that their work was generally uninterrupted by others. Comparing break behavior logged by the robot between these two groups showed that participants without clients $(n=4)$ were more likely to respond to the robot's alerts, while participants with clients $(n=2)$ were more likely to ignore them (see Figure $6 b)$. Users with clients responded to a total of 23 alerts from the robot (an average of 11.5 alerts per person) and ignored 31 alerts ( 15.5 per person), while users without clients received 90 alerts (an average of 22.5 alerts per person) and ignored 44 alerts (an average of 11 per person). Participants without clients also evaluated the robot's relative advantage $(\bar{x}=2.75>\bar{x}=1.75)$ and compatibility with their work $(\bar{x}=3.0>\bar{x}=2.0)$ more highly than did those with clients. In interviews, participants with clients remarked that the robot interrupted their work (P1, P2), and did not fit into the "rhythm" and "flow" of the office (P1, P2, P4, P10, P5, P7). Participants who did not have constant interruptions by clients (P6, P4) were more satisfied with the robot, found it more useful, and described it as providing welcomed break reminders.

In exploring the effects of the robot's social interactivity on the behavioral change and perceptions of users, our interviews suggested that the type of robot had an effect on both aspects. While only two out of four participants with alarm-type robots anthropomorphized them, participants with social robots constructed elaborate narratives about their relationships with the devices. Four out of five of the participants with social robots gave them a name. Participants in this group also personalized their robots $-\mathrm{P} 1$ accessorized hers with a scarf, while $\mathrm{P} 2$ gave her robot a face and sunglasses (see Figure 7) and said it "was a person to me." P2 talked about her experience with the prototype as if it were a relationship - at first the robot was her friend; later when it had trouble turning on it was "just like a teenager, I can't wake him up"; finally when the timing of breaks and the occasional malfunctioning of the robot became frustrating, she opined that "we were headed to counseling and possibly divorce court." P4 said she "loved [the robot] so much I wanted to keep it"; even in emails reporting technical problems she referred to the robot as "little fella" and "sweet little robot." Participants with social robots also compared them to pets: P2 saw it as standing in for an office pet she did not have, P6 compared the robot to a "fish tank," and P1 said her robot "definitely had its own personality" and was "like a little pet... a dog, a cyberdog." Even P10, who did not find the robot useful, anthropomorphized it by calling it a "creature." Some participants with alarm-type robots suggested a more social appearance would improve their experience: P9 thought a face would make the robot more cute, while P5 suggested giving it eyes.
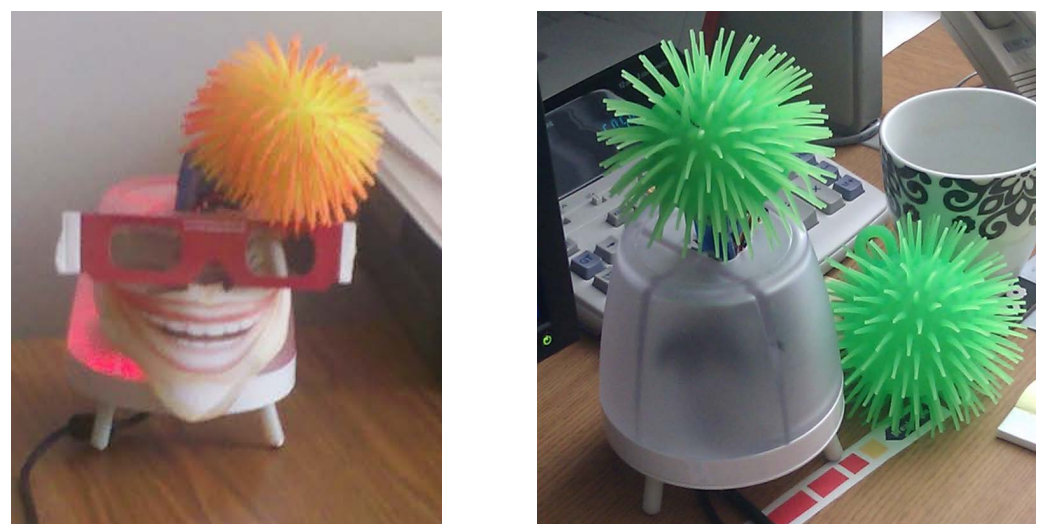

Figure 7. P2 gave her Dewey a face to make it more personable, while P4 purchased a ball that was the same as her robot to remind her of it after the study. 
Besides investing more in their relationship with the robot, participants with the social robot generally evaluated them more positively: they used 15 positive adjectives (e.g., useful, cute, novel) in the card selection exercise as compared to 6 positive attributes given by the alarm group (e.g., social, motivating, reliable). Three out of five social robot users said they would continue using the robots after the study in their interview, while none of the four alarm robot users agreed to do so. The most successful interactions happened between users who had a social robot and an uninterrupted work style - the two participants in this group rated the robot most highly in terms of effectiveness, enjoyment, and their willingness to continue using it. Our results suggest not only that very simple social cues can have an impact on how users perceive and relate to the robot, but also that a robot's behavior has to be appropriate for the user's workflow for an effective experience.

Regardless of the type of robot used, a number of participants mentioned that using the robot increased their social interactions with colleagues. The reasons given varied widely: being out of the office more (P6), the shared experience of having the robot (P9, P2), and discussing it with each other (P2, P4, and P10). This effect on social interaction among colleagues could be used more consciously as part of further design iterations. It also emphasizes the importance of studying robotic technologies within the broader social and cultural environment.

Our third design iteration displays the benefits and challenges of in situ prototype evaluation. Using a combination of self-report and behavioral measurements allowed us to identify trends in participant responses that showed the robot's sociality affected not only in regards to its efficacy but in terms of users' sense making. In the course of our study we also discovered emergent factors that should be included in the future design of robots for managing breaks-particularly in adapting to the user's workflow. This confirms previous research by Mutlu \& Forlizzi (2008) showing that work environment and interruptability are deciding factors in user evaluations of a service robot. Compared to iteration 2, we experienced less technical difficulties due to the simplified and more robust technical design, but still found that users might benefit from being reminded to think beyond the prototype's technical limitations in their evaluations.

\section{Lessons From Research Explorations with Robotic Prototypes}

Our prototype development and evaluation focused on a specific application for an office environment, and the robots we used were relatively simple in appearance and functions. More broadly, however, we presented a method of iteratively comparing contrasting design alternatives in the wild using partially functioning prototypes that is generalizable within HRI. This mode of situated research can be used to guide the development of robot forms, functions, and applications for use in various domains, particularly everyday human environments. The increasing availability of physical prototyping platforms similar to the Arduino microcontrollers we used provides a technological infrastructure that makes such research-based design exploration widely accessible to HRI researchers. This infrastructure enabled us to make inexpensive and relatively quick changes to our robot design (compared to the time it takes to design and build a fully functioning robot) and to compare variations on our design without becoming entrenched early on. Prototyping and 3D printing technologies can be used in tandem with in situ evaluation in a variety of contexts to enable people to experience interaction with robotic technologies and inform the development of contextually appropriate robotic capabilities and designs. While we developed our robot design from the ground up, our comparative and iterative approach could also be used for researching and developing contextually appropriate behaviors and applications for existing platforms, such as Rethink Robotics' Baxter, Aldebaran's NAO, telepresence robots, and others. We detail other aspects of our methods and case studies that can be of wider use in HRI below.

Our comparative semi-controlled approach to prototype development and evaluation allowed us to explore the effects of specific design factors on users' experiences of robots in the context of use and to evaluate multiple design alternatives, which inspired further iterations. By looking 
at different versions of the same design, we could understand which design factors had an effect on the interaction within the contextual dynamics of a lived-in social context. The first iteration focused on the functional requirements for the robot; the robot's embodiment gave users a chance to express preferences for the size and sound of an artifact they would be willing to use. Our comparison of an embodied robot and a desktop application in the second iteration convinced us that a physical technology would be more effective. Our findings corroborate existing research showing the importance of embodiment for persuasive robots (Bainbridge et al., 2010; Kidd \& Breazeal, 2008). Although the users of our third iteration were not all equally pleased with the robot design, their input helped us narrow down the appropriate user profile for the robots and understand the effects of robot sociality on user sense making, which can guide further robot development. The effects of sociality on people's interpretations of the robot could be extended to other robotic technologies, with an important limitation-our subjects were mostly women, which was appropriate for our context but might differ in more male-dominated or gender-balanced spaces.

As interactive probes into everyday contexts of use, our prototype evaluations also allowed us to identify emergent factors that affected users' experiences and evaluations of our robots, such as the need for the robot to fit into a particular work style and the way the robot's physical presence acted as a reminder for users to take breaks. In situ evaluations of multiple prototypes acted as "idea generators" and enabled the exploration of the technology's developing effects on user perceptions and behaviors in socially situated interactions over the course of time. User reports informed by long-term experiences with the robots described interaction breakdowns, such as the untimely break reminders given while users were busy with clients. Our participants also described new ways of using Dewey, such as the action of petting as a micro-break or the robot's presence as an inspiration for participants to socialize more with colleagues. These insights support the need to design for the HRI experience as a whole to produce design and implementation alternatives.

Our case study shows that in situ evaluations of HRI prototypes can give researchers access to users' situated experience of robot use in interactions that go on for extended periods of time. This approach allows researchers to understand not only the initial responses of potential users but also changes in their behavior and opinions beyond the novelty effect and the ways in which they adapt the technologies to their environment. Though not as robust as finished products, our prototypes also functioned as "unfinished sketches" that encouraged user feedback and enabled them to participate in the earliest stages of the design process by reinterpreting the meaning, use, and implications of robotics in their own words and on their own terms. This helped us redirect our designs based on user interpretations of the prototypes and gave users more ideas and authority when talking about potential design ideas as evaluators and critics.

In terms of data collection in semi-controlled field evaluations of prototypes, the difficulties of measuring people's perceptions and behaviors were compounded by our naturalistic environment where self-report is imprecise, direct observation is difficult or undesirable, and instrumentation can be costly and complicated. Pre- and post-surveys and self-reporting were not as informative when we wanted to track long-term behavior dynamics or understand how participants made sense of our robots. Our difficulties might have been due to the exploratory nature of our evaluations, in which we did not always know the salient factors to follow beforehand, to participants' lack of self-awareness about certain measures, and to confounding factors that affected user experiences and behaviors. Robot and sensor logs collected unobtrusively were of more value than self-reports and offset the lack of direct observational or video data; in fact, we consider them as observational data that are particularly useful in cases such as our own where the direct observation throughout the study would be very labor-intensive, partial observation might introduce bias, and video recording is not accepted by participants. Open-ended interviews were the most valuable source of information in the early stages of development, giving us an opportunity to understand the interactions from the users' points of view and helping us make sense of our other data. Triangulating between multiple data sources 
also helped us to understand a fuller picture of user experiences and the robots' functioning in context. Finally, though the small numbers of users we could handle in our in situ prototype testing did not allow for statistical analysis, the insights gained were sufficiently strong to guide further design iterations.

In situ studies are an occasion in which "the locus of control shifts from the experimenter to the participant" (Rogers, 2011, p. 59). This made our studies prone to the influence of external factors. Events like graduation week and deadlines, work rhythms, and users' responses to information about taking breaks from the news or talking to others affected how participants related to the robot. Reliance on prototype technology also left us open to technical failures, with users sometimes focusing their evaluations on prototype malfunctions rather than the design factors we were trying to investigate. As participants who had more technical experience had a higher tolerance for small breakdowns, we learned the importance of describing what to expect from test devices to participants. Our third prototype, which was the most autonomous and "finished" of the series, was also the most prone to evaluation on the basis of technical difficulties, which suggests it might be prudent to design the prototype's appearance to be commensurate with its robustness and functionality to avoid raising user expectations.

\section{Conclusion}

The case study presented here shows that in situ evaluation of robotic prototypes can provide early feedback about the usability and situated interpretation of different aspects of robotic design (e.g., form, function) in the context of use that can inform the design and science of HRI. Our researchbased design approach was inspired by a desire to experiment with form, function, and interactivity while involving user input in robot development as early and often as possible. Despite implementation challenges, prototyping and evaluation allowed us to discover the everyday problems that our participants needed solved. We also assessed the effects of specific design factors, such as embodiment and social interactivity, on the performance of our prototype through the implementation of multiple possible design solutions. We found it fruitful to focus our design activities on a particular space and task, as it helped us learn more through successive prototyping and evaluation iterations. Field evaluations challenged our design assumptions, gave new directions to our design activities, and informed us about unexpected factors that had significant effects on the ways users experienced and evaluated our robots. Prototype evaluation in the field also left various aspects of the studies out of our control, which led to both malfunctions and unexpected findings during our field trials and also encouraged us to reflect on our design assumptions and goals.

\section{Acknowledgements}

We are grateful to all the participants in our studies for their time, patience, and insights. We would also like to thank Matthew Francisco, Kevin Walorski, Leo Pak, Daryl Hansen, Kurt Weisman, Lorelei Kelly, Zachary Zimmerman, Ebony Holly, and other members of our lab for their assistance in this project.

\section{References}

Alač, M., Movellan, J., \& Tanaka, F. (2011). When a robot is social: Spatial arrangements and multimodal semiotic engagement in the practice of social robotics. Social Studies of Science, 41(6), 893-926. doi:10.1177/0306312711420565

Bainbridge, W. A., Hart, J. W., Kim, E. S., \& Scassellati, B. (2010). The benefits of interactions with physically present robots over video-displayed agents. International Journal of Social Robotics, 3(1), 41-52. doi: 10.1007/s12369-010-0082-7 
Bartneck, C., \& Hu, J. (2004). Rapid prototyping for interactive robots. In The 8th Conference on Intelligent Autonomous Systems (IAS-8), pp. 136-145. Retrieved from http://www.idemployee.id.tue.nl/j.hu/publications/RapidPrototyping_ias8/RapidPrototyping_ias8. html

Bethel, C. L., \& Murphy, R. R. (2008). Survey of non-facial/non-verbal affective expressions for appearance-constrained robots. IEEE Transactions on Systems, Man, and Cybernetics, Part C: Applications and Reviews, 38(1), 83-92. doi:10.1109/TSMCC.2007.905845

Bijker, W. E. (1995). Sociohistorical technology studies. In Jasanoff, S., Markle, G. E., Petersen, J. C., Pinch, T. (eds.). Handbook of Science and Technology Studies. Thousand Oaks: Sage Publications, pp. 9-256.

Bird, J., Marshall, P., \& Rogers, Y. (2009). Low-fi skin vision: A case study in rapid prototyping a sensory substitution system. In Proceedings of the 23rd British HCI Group Annual Conference on People and Computers: Celebrating People and Technology (pp. 55-64). Swinton, UK: British Computer Society. Retrieved from http://dl.acm.org/citation.cfm?id=1671011.1671018

Broekens, J., Heerink, M., \& Rosendal, H. (2009). Assistive social robots in elderly care: A review. Gerontechnology, 8(2), pp. 94-103. doi:10.4017/gt.2009.08.02.002.00

Buchenau, M., \& Suri, J. F. (2000). Experience prototyping. In Proceedings of the 3rd conference on Designing interactive systems: Processes, practices, methods, and techniques (pp. 424-433). New York: ACM. doi:10.1145/347642.347802

Chang, W. L., Šabanović, S., \& Huber, L. (2013). Situated analysis of interactions between cognitively impaired older adults and the therapeutic robot PARO. In Proceedings of $5^{\text {th }}$ International Conference of Social Robotics (ICSR 2013), Bristol UK, October 27-29 (pp. $371-$ 380). doi: 10.1007/978-3-319-02675-6_37

Dautenhahn, K. (2007). Methodology and themes of human-robot interaction: A growing research field. Advanced Robotic Systems, 4(1), 103-108. Retrieved from http://uhra.herts.ac.uk/handle/2299/3814

Davis, F. D. (1993). User acceptance of information technology: System characteristics, user perceptions, and behavioral impacts. International Journal of Man-Machine Studies, 38(3), 475487.

DiSalvo, C., Lodato, T., Fries, L., Schechter, B., \& Barnwell, T. (2011). The collective articulation of issues as design practice. CoDesign, 7(3-4), 185-197. doi:10.1080/15710882.2011.630475

DiSalvo, C., Nourbakhsh, I., Holstius, D., Akin, A., \& Louw, M. (2008). The Neighborhood Networks project: A case study of critical engagement and creative expression through participatory design. In Proceedings of the Tenth Anniversary Conference on Participatory Design 2008 (pp. 41-50). Indianapolis, IN: Indiana University. Retrieved from http://dl.acm.org/citation.cfm?id=1795234.1795241

Doherty, R. W. (1997). The emotional contagion scale: A measure of individual differences. Journal of Nonverbal Behavior, 21, 131-154. doi:10.1023/A:1024956003661

Dow, S. P., Glassco, A., Kass, J., Schwartz, M., Schwartz, D., \& Klemmer, S. (2010). Parallel prototyping leads to better design results, more divergence, and increased self-efficacy. $A C M$ Transactions on Computer-Human Interaction, 17(4), 18:1-18:24. doi:10.1145/1879831.1879836

Dow, S. P., Fortuna, J., Schwartz, D., Altringer, B., Schwartz, D. L., Klemmer, S. (2011). Prototyping dynamics: Sharing multiple designs improves exploration, group rapport, and results. $\mathrm{Ub}$ Proceedings of the ACM CHI Conference on Human Factors in Computing Systems (CHI 2011) (pp. 2807-2816). New York: ACM. doi:10.1145/1978942.1979359 
Forlizzi, J. (2007). How robotic products become social products: An ethnographic study of cleaning in the home. In Proceedings of the ACM/IEEE International Conference on HumanRobot Interaction (pp. 129-136). New York: ACM. doi:10.1145/1228716.1228734

Forlizzi, J., DiSalvo, C., \& Gemperle, F. (2004). Assistive robotics and an ecology of elders living independently in their homes. Human-Computer Interaction, 19(1), 25-59.

doi:10.1207/s15327051hci1901\&2_3

Gates, W. (2007). A Robot in Every Home. Scientific American, 296(1), 58-65. Retrieved from http://www.scientificamerican.com/article.cfm?id=a-robot-in-every-home

Gould, J. D., \& Lewis, C. (1985). Designing for usability: Key principles and what designers think. Communications of ACM, 28(3), 300-311. doi:10.1145/3166.3170

Hakken, D., Teli, M., \& D'Andrea, V. (2010). Intercalating the social and the technical: Socially robust and enduring computing. In Proceedings of the $11^{\text {th }}$ Biennial Participatory Design Conference (PDC '10). New York: ACM.

Hazlewood, W. R., Stolterman, E., \& Connelly, K. (2011). Issues in evaluating ambient displays in the wild: Two case studies. In Proceedings of the SIGCHI Conference on Human Factors in Computing Systems (pp. 877-886). New York: ACM. doi:10.1145/1978942.1979071

Holmquist, L. E. (2005). Prototyping: Generating ideas or cargo cult designs? Interactions, 12(2), 48-54. doi:10.1145/1052438.1052465

Inoue, K., Wada, K., \& Ito, Y. (2008). Effective application of Paro: Seal type robots for disabled people in according to ideas of occupational therapists. Paper presented at the 11th International Conference on Computers Helping People with Special Needs, Linz, Austria.

Jafarinaimi, N., Forlizzi, J., Hurst, A., \& Zimmerman, J. (2005). Breakaway: An ambient display designed to change human behavior. In CHI '05 Extended Abstracts on Human Factors in Computing Systems (pp. 1945-1948). New York: ACM. doi:10.1145/1056808.1057063

Jett, Q. R., \& George, J. M. (2003). Work interrupted: A closer look at the role of interruptions in organizational life. Academy of Management Review, 28(3), 494-507. doi:10.5465/AMR.2003.10196791

Kanda, T., Sato, R., Saiwaki, N., \& Ishiguro, H. (2007). A two-month field trial in an elementary school for long-term human-robot interaction. IEEE Transactions on Robotics, 23(5), 962-971. doi:10.1109/TRO.2007.904904

Kanda, Takayuki, Shiomi, M., Miyashita, Z., Ishiguro, H., \& Hagita, N. (2009). An affective guide robot in a shopping mall. In Proceedings of the 4th ACM/IEEE International Conference on Human-Robot Interaction (pp. 173-180). New York: ACM. doi:10.1145/1514095.1514127

Kidd, C. D., \& Breazeal, C. (2008). Robots at home: Understanding long-term human-robot interaction. In IEEE/RSJ International Conference on Intelligent Robots and Systems (IROS 2008) (pp. 3230-3235). doi:10.1109/IROS.2008.4651113

Kozima, H., Michalowski, M. P., \& Nakagawa, C. (2009). Keepon. International Journal of Social Robotics, 1(1), 3-18. doi:10.1007/s12369-008-0009-8

Lee, H., Sung, J., Šabanović, S., \& Han, J. (2012). Cultural design of domestic robots: A study of user expectations in Korea and the United States. Proceedings of the IEEE International Symposium on Robot and Human Interactive Communication (RO-MAN 2012) (pp. 803-808), Paris, France.

Lee, M. K., Forlizzi, J., Rybski, P. E., Crabbe, F., Chung, W., Finkle, J., ... Kiesler, S. (2009). The Snackbot: Documenting the design of a robot for long-term human-robot interaction. In 
Proceedings of the 4th ACM/IEEE International Conference on Human Robot Interaction (pp. $7-$ 14). New York: ACM. doi:10.1145/1514095.1514100

Liu, L. \& Maddux, C. (2008). Technology Attitude Instrument. Computers in the Schools, 25, $145-158$.

Lim, Y.-K., Stolterman, E., \& Tenenberg, J. (2008). The anatomy of prototypes: Prototypes as filters, prototypes as manifestations of design ideas. ACM Transactions on Computer-Human Interaction, 15(2), 7:1-7:27. doi:10.1145/1375761.1375762

Michalowski, M. P., Šabanović, S., \& Simmons, R. (2006). Roillo: Creating a social robot for playrooms. In Proceedings of the IEEE International Symposium on Robot and Human Interactive Communication (RO-MAN 2006) (pp. 587-592).

Michalowski, M. P., Šabanović, S., Disalvo, C., Busquets, D., Hiatt, L. M., Melchior, N. A., \& Simmons, R. (2007). Socially Distributed Perception: GRACE plays social tag at AAAI 2005. Autonomous Robots, 22(4), 385-397. doi:10.1007/s10514-006-9015-6

Moore, G. C. and Benbasat, I. 1991. Development of an instrument to measure the perceptions of adopting an information technology innovation. Information Systems Research, 2(3), 192-222.

Morris, D., Brush, A. B., \& Meyers, B. R. (2008). Superbreak: Using interactivity to enhance ergonomic typing breaks. In Proceedings of CHI'08 (pp. 1817-1826). New York: ACM. doi: $10.1145 / 1357054$

Mutlu, B., \& Forlizzi, J. (2008). Robots in organizations: The role of workflow, social, and environmental factors in human-robot interaction. In Proceedings of the $3 r d$ ACM/IEEE International Conference on Human-Robot Interaction (pp. 287-294). New York: ACM. doi:10.1145/1349822.1349860

Nomura, T., Tasaki, T., Kanda, T., Shiomi, M., Ishiguro, H., \& Hagita, N. (2007). Questionnairebased social research on opinions of Japanese visitors for communication robots at an exhibition. AI \& SOCIETY, 21(1-2), 167-183. doi:10.1007/s00146-006-0053-6

Nomura, T., Kanda, T., \& Suzuki, T. (2006). Experimental investigation into influence of negative attitudes toward robots on human-robot interaction. AI \& Society, 20(2), 138-150.

Nye, D. E. (2007). Technology Matters: Questions to Live With. Cambridge MA: The MIT Press.

Reeder, S., Kelly, L., Kechavarzi, B., \& Šabanović, S. (2010). Breakbot: A social motivator for the workplace. In Proceedings of the 8th ACM Conference on Designing Interactive Systems (pp. 61-64). New York: ACM. doi:10.1145/1858171.1858184

Rettig, M. (1994). Prototyping for tiny fingers. Communincations of ACM, 37(4), 21-27. doi:10.1145/175276.175288

Riek, L. D. (2012). Wizard of Oz studies in HRI: A systematic review and new reporting guidelines. Journal of Human-Robot Interaction, 1(1), 119-136. Retrieved from http://www.humanrobotinteraction.org/journal/index.php/HRI/article/view/9

Rogers, Y. (2011). Interaction design gone wild: Striving for wild theory. Interactions, 18(4), 58 62. doi:10.1145/1978822.1978834

Šabanović, S., Michalowski, M. P., \& Simmons, R. (2006). Robots in the wild: Observing humanrobot social interaction outside the lab. In Proceedings of the 9th IEEE International Workshop on Advanced Motion Control, 2006 (pp. 596-601). Presented at the 9th IEEE International Workshop on Advanced Motion Control, 2006. doi:10.1109/AMC.2006.1631758

Šabanović, S., Reeder, S., Kechavarzi, B., \& Zimmerman, Z. (2011). Designing a robot through 
prototyping in the wild. In Proceedings of the 6th International Conference on Human-Robot Interaction (pp. 239-240). New York: ACM. doi:10.1145/1957656.1957750

Šabanović, S. (2010). Robots in society, society in robots: Mutual shaping of society and technology as a framework for social robot design. International Journal of Social Robotics, 2(4), 439-450.

Suchman, L. (2007). Human-Machine Reconfigurations: Plans and Situated Actions. Cambridge: Cambridge University Press.

Sung, J., Christensen, H. I., \& Grinter, R. E. (2009). Robots in the wild: Understanding long-term use. In 2009 4th ACM/IEEE International Conference on Human-Robot Interaction (HRI) (pp. 45-52). doi: 10.1145/1514095.1514106

Tanaka, F., Cicourel, A., \& Movellan, J. R. (2007). Socialization between toddlers and robots at an early childhood education center. Proceedings of the National Academy of Sciences, 104(46), 17954-17958. doi:10.1073/pnas.0707769104

Tapus, A., Mataric, M. J., \& Scasselati, B. (2007). Socially assistive robotics [Grand Challenges of Robotics]. IEEE Robotics Automation Magazine, 14(1), 35-42. doi:10.1109/MRA.2007.339605

Turkle, S. (2006). A nascent robotics culture: New complicities for companionship. AAAI Technical Report Series, July 2006. Retrieved from http://web.mit.edu/sturkle/www/pdfsforstwebpage/ST_Nascent\%20Robotics\%20Culture.pdf

Vertesi, J. (2012). "Seeing like a rover": Visualization, embodiment and interaction on the Mars Exploration Rover Mission. Social Studies of Science, 42(3), 393-414. doi: $10.1177 / 0306312712444645$

Wada, K., \& Shibata, T. (2007). Living with seal robots-Its sociopsychological and physiological influences on the elderly at a care house. IEEE Transactions on Robotics, 23(5), 972-980. doi:10.1109/TRO.2007.906261

Authors' names and contact information: S. Šabanović, Indiana University, Bloomington, USA. Email: selmas@indiana.edu; S. M. Reeder, Carnegie Mellon University, Pittsburgh, USA. Email: smreeder@gmail.com; B. Kechavarzi, Indiana University-Purdue University, Indianapolis, USA. Email: bkechava@indiana.edu 